\title{
Fertility after Neosalpingostomy through Laparoscopic Way in Point "G" Teaching Hospital
}

\author{
Kéita Soumaila1 ${ }^{*}$, Traoré Youssouf ${ }^{2}$, Soumaré Lamine', Koumaré Sekou${ }^{1}$, Sacko Oumar1, \\ Camara Aboubacar' ${ }^{1}$, Camara Moussa ${ }^{1}$, Sissoko Moussa1, Abdoulaye Sissoko', Koita Adama1, \\ Sanogo Z. Zimogo ${ }^{1}$ \\ ${ }^{1}$ Surgery Department “A” Point G Teaching Hospital, Bamako, Mali \\ ${ }^{2}$ Faculty of Medicine and Odonto Stomatology of Bamako, University of Science, Technique and Technologies of Bamako \\ (USTTB), Bamako, Mali \\ Email: ^keita_soumi@yahoo.fr, ^dryoussouf.traore@gmail.com
}

How to cite this paper: Soumaila, K., Youssouf, T., Lamine, S., Sekou, K., Oumar, S., Aboubacar, C., Moussa, C., Moussa, S., Sissoko, A., Adama, K. and Zimogo, S.Z. (2019) Fertility after Neosalpingostomy through Laparoscopic Way in Point "G" Teaching Hospital. Surgical Science, 10, 49-58.

https://doi.org/10.4236/ss.2019.102007

Received: December 25, 2018

Accepted: February 15, 2019

Published: February 18, 2019

Copyright $\odot 2019$ by author(s) and Scientific Research Publishing Inc. This work is licensed under the Creative Commons Attribution International License (CC BY 4.0).

http://creativecommons.org/licenses/by/4.0/

\begin{abstract}
The endoscopic surgery is actually the golden standard for the management of infertility. Its results look better than classical surgery. The aim of the study was to assess the fertility rate among infertile women who have undergone a laparoscopic distal tubal plasty and to estimate factors influencing this fertility. From January 2007 to December 2016 a descriptive and analytical cross sectional retrospective study was conducted in the departments of general and laparoscopic surgery of the Point "G" Teaching Hospital in Bamako, Mali. Clinical records of 120 laparoscopic patients with distal tubal occlusion who have undergone a neosalpingostomy were collected. Average period of infertility was 6.7 years (from 1 to 25 years), and the average duration before laparoscopic surgery was 5 years. Main abnormalities were: 50 cases of bilateral tubal distal occlusion (41.7\%), 14 patients with unilateral tubal distal occlusion (11.7\%), 44 cases of bilateral hydrosalpinx (36.6\%) and 12 cases with unilateral occlusion (10.0\%). Seventeen patients had adhesions (14.1\%) including $4.0 \%$ of tubo-peritoneal adhesions, $35.5 \%$ of tubo-ovary adhesions and Fitz Hugh and Curtis syndrom adhesions in $17.7 \%$ of cases. More than half of the patients (58.0\%) had a grade II tubal score. Neosalpingostomy had led to bilateral tubal repermeabilization among $60.0 \%$ of the patients. Thirty two point five percent (39/120) pregnancies have been observed after the management. Conception probability was null after the $4^{\text {th }}$ post operative year. The majority of pregnancies have been observed in the $11^{\text {th }}$ month after laparoscopic management. During the follow-up, thirty one patients had intrauterine pregnancy $(79.5 \%)$, four ectopic pregnancies and three miscarriages. The occurrence of pregnancy was influenced by the desire of the
\end{abstract}


woman, the absence of co-morbidity, the tubal score, the result and the quality of the laparoscopic gesture and the duration of the fertility. Conclusion: In our working setting, neosalpingostomy by laparoscopic surgery is an accessible and feasible method in the management of distal tubal infertility. It has permit to treat infertile patients with more chance of success. The occurrence of pregnancy is associated to tubal disease thoroughness, endoscopic gestures quality and the duration of the fertility.

\section{Keywords}

Fertility, Laparoscopy, Neosalpingostomy, Pregnancy

\section{Introduction}

It is well known that normal reproduction requires a finely orchestrated concert of hypothalamic-pituitary-ovarian-tubal-uterine events [1]. Infertility of a woman constitutes a problem especially in Africa. The distal portion of the oviduct is essential for the initiation of ovum transport and support. The fimbriae play a unique and highly intricate role in ovum capture and travel.

The part of a woman in infertility cause is estimated to $30 \%$ [2]. Distal fallopian pathology is found among $40 \%-50 \%$ of infertile patients [3]. In Mali, it represents $50 \%-80 \%$ of female infertility cause [4].

When the distal fallopian tube has been altered, such as in pelvic inflammatory disease, attempts to surgical treatment have been traditionally associated with poor results. Neosalpingostomy (salpingostomy) is the surgical management of the distal occlusion and the creation of a tubal ostium. Long-term follow-up studies of patients who have undergone neosalpingostomy are sparse [5].

Surgery of the oviduct for infertility has given such disappointing results that it is regarded with disfavor, and many gynecologists do not practice it at all. The discouraging results of this surgery have shown some advance over the years. [6]. However in our area, it constitutes the main method to treat patients with tubal obstruction.

Endoscopic surgery introduced in Mali in 2001, is in our work setting the main method used to treat distal tubal infertility. Although its results are often poor [5] [6], it permits a curative treatment of tubal occlusion and offers to the patient the possibility to expect babies without using medical assisted procreation that is hardly available in Mali.

The purpose of this analytical and cross sectional retrospective study was to assess the fertility rate among infertile women who have undergone a laparoscopic distal tubal plasty and also to estimate factors influencing this fertility.

\section{Material and Method}

\subsection{Study Setting}

Point "G" teaching hospital is a tertiary care referral center in Bamako, Mali. It is 
affiliated with the Faculty of Medicine and Odonto-stomatology at the University of Bamako. This hospital has been providing endoscopic cares since 2001. The management of infertile patients is done in collaboration with other services such gynecology and obstetrics, laboratory of the faculty and urological department.

\subsection{Type of Survey and Sampling}

It was a descriptive and analytical cross sectional retrospective study that took place in the departments of general surgery and laparoscopic of Point " $G$ " teaching hospital from 2007 to 2016.

Inclusion criteria: all the cases of endoscopic surgery used to treat distal tubal infertility, absence of other fertility factors and semen abnormalities.

Non-inclusion criteria: cases of classical surgery used for distal tubal occlusion treatment and patients with other causes of infertility.

\subsection{Procedures}

Before operation, patients were evaluated with semen analysis, basal body temperature charts, post-coital test, and timed endometrial biopsy specimen to rule out other possible causes of infertility. Hysterosalpingography and a laparoscopy were also performed to document distal occlusion once tubal disease was suspected. Patients were selected for neosalpingostomy based on the preliminary finding of distal disease, confirmed by hysterosalpingography.

Each patient received Decapeptil by injection way, and doxycycline or amoxicillin 4 hours before the procedure and amoxicillin by oral way during seven days after the procedure.

The equipment used was a laparoscopic column placed at the foot of the table with mostly reusable devices. All patients were operated under general anesthesia.

Distal tubal involvement was suspected by using ultrasound and hysterosalpingography, and confirmation was made on the basis of macroscopic laparoscopic findings.

Tubal permeability was tested before and after the procedure, by injecting blue methylen solution through the intra-uterine device.

The fallopian was opened by a cross incision on the hydrosalpinx. The opening of the new fimbrial of the oviduct was maintained by using unipolar or bipolar coagulation.

The average duration of follow-up was 4 years with extremes of one year and 7 years.

The information of patients was collected by phone call or home visit.

Criterion of judgment was occurrence of pregnancy among patients who have received the endoscopic treatment.

Data were collected from surgical reports, admissions records, and prenatal care documents.

Statistical Package for the Social Sciences (Spss) 16.0 was used for data analysis. Comparison of qualitative variables was made by using the chi square test or 
Fisher exact test, the quantitative variables comparison was done by Student's $t$ test. Logistic regression has been also used to appreciate factors that have influenced the fertility among patients who have undergone laparoscopic distal tubal plasty. Results have been expressed in percentage with their Odd ration (OR), $95 \%$ confidence interval $\left(\mathrm{CI}_{95}\right)$. P value $<1 \%$ considered significant.

\section{Ethical Aspect}

Confidentiality has been respected for all the patients.

\section{Results}

One hundred and twenty clinical files have been collected according to inclusion criteria of the study. The rate of married women was $98.0 \%$. Patients aged from 31 to 40 years were $81.0 \%$ with extremes ages of 17 years and 47 years. The average duration of infertility was 6.7 years with deviation type of 4.39. Minimal duration of infertility was one year and the maximum was 25 years. It was about primary infertility in $40.9 \%$ of cases $(\mathrm{n}=49)$ and secondary infertility in $59.1 \%$ of cases. Patients have been treated first by a gynecologist in $92.1 \%$. Eighty five percent $(85.0 \% ; \mathrm{n}=102)$ have been referred by a gynecologist versus $15.0 \%$ who have come by themselves in our service.

Hysterosalpingography has shown 50 cases of bilateral tubal distal occlusion (41.7\%), 14 patients with unilateral tubal distal occlusion (11.7\%), 44 cases of bilateral hydrosalpinx (36.6\%) and 12 cases with unilateral occlusion (10.0\%). Seventeen patients had adhesions (14.1\%) including $4.0 \%$ of tubo-peritoneal adhesions, 35.5\% of tubo-ovary adhesions and Fitz Hugh and Curtis syndrom adhesions in $17.7 \%$ of cases.

More than half of the patients (58.0\%) had a grade II tubal score. Neosalpingostomy had led to bilateral tubal repermeabilization among $60.0 \%$ of the patients.

Thirty-two point five percent of pregnancies (39/120) have been observed after the management.

Conception probability was null after the fourth year post endoscopic management.

Main results of macroscopic abnormalities during endoscopic management are noted in Table 1. The endoscopic treatment undertaken is summarized in Table 2.

Tuba disease score according Donnez classification is: grade I (5.0\%), grade II (58.0\%), grade III (33.00) and grade IV (4.0\%).

After endoscopic management, bilateral fallopian repermeabilization has been obtained in 72 patients $(60.0 \%)$.

The majority of pregnancies has been observed in $11^{\text {th }}$ month after laparoscopic management with extremes of 2 months and 48 months. Fecundity has been observed in $32.5 \%(n=39)$.

The main results of the pregnancy evolution were: 31 births (79.5\%), 1 ongoing pregnancy, 4 ectopic pregnancies and 3 miscarriages. Table 2 shows the re- 
sults of fecundity according to endoscopic management types.

In multivarious analyze, Table 3 shows factors that have influenced the fertility among patients who have undergone a laparoscopic distal tubal plasty.

Main coast of the endoscopic management was 354 dollars US.

Table 1. Characteristics of patients and types of tubal diseases that have been observed during endoscopic management.

\begin{tabular}{|c|c|c|}
\hline \multicolumn{3}{|c|}{ Characteristics of patients $(n=72)$} \\
\hline Previous characteristic of patients & $N$ & $\%$ \\
\hline Repeated genital infection & 48 & 66.7 \\
\hline Pelvic surgery* & 13 & 18 \\
\hline Miscarriage & 4 & 5.6 \\
\hline \multicolumn{3}{|c|}{ Macroscopic aspects of the fallopian $(n=120)$} \\
\hline Falloppia aspects & $N$ & $\%$ \\
\hline Hydrosalpinx & 74 & 61.7 \\
\hline Phimosys & 12 & 10 \\
\hline Adhesions & 17 & 14.1 \\
\hline Serious Recovery & 9 & 7.5 \\
\hline Muquous thickening of the salpinx folds & 8 & 6.7 \\
\hline
\end{tabular}

*Three patients had previous Cesarean, two cases of ectopic pregnancy, Four cases of myomectomy, one case of Kystectomy, three cases of tubal plasty.

Table 2. Relation between intervention types and fecundity of patients after endoscopic management $(\mathrm{n}=120)$.

\begin{tabular}{ccccccc}
\hline & \multicolumn{5}{c}{ Fecundity of patients after endoscopic management } \\
\cline { 2 - 6 } Intervention types & $\begin{array}{c}\text { Birth } \\
\mathrm{n}(\%)\end{array}$ & $\begin{array}{c}\text { Ectopic } \\
\text { pregnancy } \mathrm{n}(\%)\end{array}$ & $\begin{array}{c}\text { Miscarriage } \\
\mathrm{n}(\%)\end{array}$ & $\begin{array}{c}\text { Ongoing } \\
\text { pregnancy n (\%) }\end{array}$ & $\begin{array}{c}\text { No } \\
\text { pregnancy }\end{array}$ & $\begin{array}{c}\text { Total }(\%) \\
\mathrm{n}(\%)\end{array}$ \\
\hline Neosalpingostomy & $18(15.0 \%)$ & $2(1.7 \%)$ & $2(1.7 \%)$ & $1(0.8 \%)$ & $42(35.0 \%)$ & $65(54.2 \%)$ \\
Neosalpingostomy + Lysis of adhesions & $2(1.7 \%)$ & $1(0.8 \%)$ & $0(0.0 \%)$ & $0(0.0 \%)$ & $3(2.5 \%)$ & $6(5.0 \%)$ \\
Neosalpingostomy + Fimbrioplasty & $1(0.8 \%)$ & $0(0.0 \%)$ & $0(0.0 \%)$ & $0(0.0 \%)$ & $2(1.7 \%)$ & $3(2.5 \%)$ \\
Fimbrioplasty & $1(0.8 \%)$ & $1(0.8 \%)$ & $0(0.0 \%)$ & $0(0.0 \%)$ & $5(4.2 \%)$ & $7(5.8 \%)$ \\
Lysis of adhesions & $8(6.7 \%)$ & $0(0.0 \%)$ & $1(0.8 \%)$ & $0(0.0 \%)$ & $28(23.3 \%)$ & $37(30.8 \%)$ \\
Fimbrioplasty + Lysis of adhesions & $1(0.8 \%)$ & $0(0.0 \%)$ & $0(0.0 \%)$ & $0(0.0 \%)$ & $1(0.8 \%)$ & $2(1.7 \%)$ \\
Total & $\mathbf{3 1 ( 2 5 . 8 \% )}$ & $\mathbf{4 ( 3 . 3 \% )}$ & $\mathbf{3 ( 2 . 5 \% )}$ & $\mathbf{1 ( 0 . 8 \% )}$ & $\mathbf{8 1 ( 6 7 . 5 \% )}$ & $\mathbf{1 2 0 ( 1 0 0 . 0 \% )}$ \\
\hline
\end{tabular}

Fisher test $=23,698$ and $\mathrm{p}$ value $=0.0001$.

Table 3. Factors influencing the fertility among patients who have undergone a laparoscopic distal tubal plasty from 2007 to 2016 in Point "G" teaching hospital (multivarious analysis).

\begin{tabular}{llc}
\hline \multicolumn{1}{c}{ Factors } & OR & $\mathrm{CI}_{95 \%}$ \\
\hline Donnez Tubal score $<$ III and IV & 2.33 & {$[0.02-3.96]$} \\
The absence of co-morbidity & 7.00 & {$[1.00-12.94]$} \\
Neosalpingostomy & 5.00 & {$[3.66-10.10]$} \\
Lysis of adhesions & 4.01 & {$[1.04-12.94]$} \\
Period between endoscopic management and conception $\leq 4$ years & 0.11 & {$[0.01-2.91]$} \\
Duration of infertility $>$ 10 years & 1.20 & {$[0.96-3.07]$} \\
Follow-up by a gynecologist & 2.66 & {$[1.77-6.00]$} \\
\hline
\end{tabular}




\section{Discussion}

As any retrospective studies, our survey has some limits that are incomplete data of patients. However this survey keeps its epidemiological and clinical interest. Statistical analysis has reduced information and selection biases.

The non availability of medical assisted procreation accounts for the rate of endoscopic management in our service despite the high cost of this intervention. The majority of our patients were between 31 and 40 years. In our study the youngest was 17 years and the oldest was 47 years. That can be explained by the desire to get a baby before the menopause for all of them. Most of them after wedding stay many years without conception and they undertook visit to satisfy this social desire. That is the reason why more than half of our patients have undertaken early infertility visit.

In Mali, fallopian tubal diseases are the main cause of infertility [4]. Their frequency varies from $50 \%$ to $80 \%$.

Before endoscopic management most of our patients have received a treatment from a gynecologist first. They required endoscopic management after failure of this treatment. Such management was not available also in other public services until 2014

Most of our patients had repeated genital infection and had undergone pelvis surgery (Table 1) that have altered the functionality of the tubal uterus.

Laparoscopy permits to assess tubal diseases and to reveal a significant number of tubal lesions not detected by hysterosalpingography. It also permits the reconstitution of a functional oviduct, allows to hope rates of intrauterine pregnancy in $30 \%$ of cases [7]. Laparoscopic exploration revealed a hydrosalpinx in $61.7 \%$ and $14.1 \%$ of adhesions (Figure 1 and Figure 2). The prevalence of tubal adhesions in infertile women varies between 10 and 23\% [8].
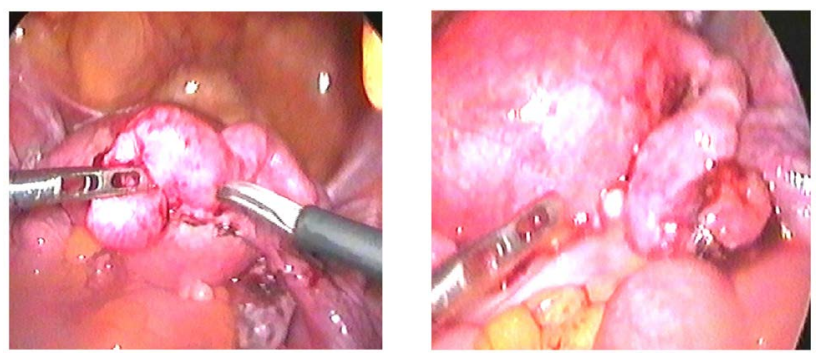

Figure 1. Incision of hydrosalpinx (left image) and neosalpingostomy after fimbrial tubal reversion (right image).

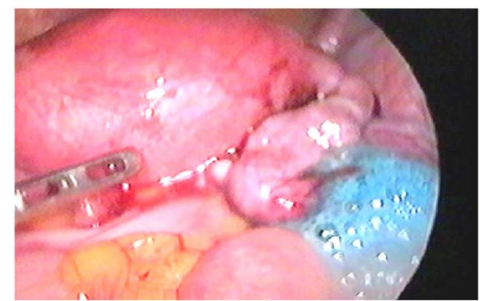

Figure 2. Positive blue methylen test. 
This restoration of tubal permeability was necessary but not sufficient in the occurrence of spontaneous pregnancies. Like Kaoulher [9], we have obtained bilateral restoration of the tubal permeability among more than half of the patients. Negative predictive factors have been implicated in the chances of conception, including the presence of adhesions, poor quality of tubal fringes and mucosal folds [10]. According to Boer-Meisel [10], a tubal score of I or II improves pregnancy chance approximatively from $40 \%$ to $50 \%$, but less than $5 \%$ when the tubal score is III. The tubal score established in 64 patients in our study was grade I in 5\%, Grade II in 58\%, Grade III in 33\% and Grade IV 4\%. This result can explain the rate of occurring o pregnancy in our study.

The return to fertility was observed in 39 patients (32.5\%). For Jeffrey [5], the initial evaluation of the 72 patients who underwent bilateral neosalpingostomy revealed a rate of $27.7 \%$ intrauterine pregnancy. Gomel and McComb [11] found $60 \%$ of successful term pregnancies occurred after the first postoperative year. They also reported two unexpected pregnancies in patient with proven distal occlusion who did not undergo corrective surgery, suggesting possible drainage and regeneration of the infundibular portion of the tube. [11]. Verhoeven [12] reported after microsurgical terminal salpingostomy $19.6 \%$ patients who delivered, $3.6 \%$ spontaneous abortions and $1.8 \%$ ectopic pregnancies. In the survey carried out by Sanogo [13] fertility rate was $38.5 \%$ and divided as follows: live childbirth (56.7\%), spontaneous abortions (18.3\%), current pregnancy (13.3\%), ectopic pregnancy $(6.7 \%)(\mathrm{P}=0.001)$. Margit $[14]$ reported the term pregnancy and intrauterine pregnancy rates after salpingostomy of $22 \%$ and $29 \%$, respectively. Our ectopic pregnancy rate as more than four time high than DeCherney [15]. The chance of conception was null after the fourth endoscopic postoperative year, which could be related to irreversible and permanent subfertility factors. Patients between the ages of 21 and 25 years had a better prognosis, with $27.9 \%$ having full-term pregnancies. Successful pregnancies in these studies probably reflect patients in whom tubal physiology was slowly restored [11]. These results confirm that the successful of endoscopic management indicated to treat infertile patients are variable and depends on many factors [16] [17] [18] [19] [20].

Poor results may be related to the insufficient time allotted for patient follow-up examination and for intrinsic restoration of secretory and ciliary function to return to the fallopian tube. [5]. Conception after laparoscopic surgery was favorably influenced by the desire to procreate, the absence of co-morbidity, the tubal score, the quality of the surgical procedure and the subsequent follow-up by a gynecologist (Table 3). The results indicate that three factor scores are associated with (almost) complete pregnancy failure: many adhesions [21], fixed adhesions, and a thick tubal wall. Pregnancy success is mainly related to a normal endosalpinx and to the absence of adhesions [11] [22].

None of the remaining scores appear to differentiate between very high and very low pregnancy rates. Abortion rates show the same tendency as term pregnancy rates. No clear pattern appears to be present for the ectopic preg- 
nancies.

The salvage rate of live babies following plastic surgery for blocked tubes showed a considerable variation, depending on whether it was calculated from the results of all surgeons, or of two surgeons, or from those of one surgeon working on private patients only, selected by himself. When the salvage rate was calculated from the results of all surgeons, there was one live baby born for every $61 / 2$ operations for blocked tubes (excluding salpingolysis). However, the live birth rate for one surgeon working on private patients only was one baby for every three and half operations. These results emphasise the importance of selecting patients for operation who have no infertility factors present other than the blocked tubes. In the group operated on by "all surgeons," there were women with subfertile husbands or severe ovulatory defects, and even some whose total work-up was limited to a tubal investigation only [6].

Despite our encourageous results, we think the success of neossalpingostomy in infertile patients is related to the time after surgery. Indeed, the increase in the pregnancy rate may reflect the ability of the fallopian tube for ciliogenesis and intrinsic repair. Thus, if patients have not conceived within 2 years of neosalpingostomy for distal tubal obstruction, significant reproductive potential still exists [5].

We finally think as Margit [14], Donnez [23] and Gomel, V. (1980) [24] that the pregnancy outcome after tubal surgery is related to many variables reflecting the extent and severity of preexisting disease. Some of the factors reported in the literature are as follows: the nature and extent of adhesions, [1] [11] [21] the extent of tubal damage [17], the macroscopic condition of the endosalpinx, the microscopic appearance of the ciliary epithelium [16], the presence of intratubal adhesions [11] [16], the status of the tubal wall, [18] and the presence of a rugal pattern in the tubes of the preoperative hysterosalpingogram [1]. These factors were shown to be associated with the prognosis of the patient with regard to the achievement of an intrauterine pregnancy.

\section{Conclusion}

Neosalpingostomy by laparoscopic surgery is a method of management that improves conception chance of infertile women. However many factors can influence the conception after this method. These are tubal disease thoroughness, endoscopic gestures quality and the duration of the fertility. A best selection of patients according to these factors should improve the success rate of women.

\section{Conflicts of Interest}

The authors declare no conflicts of interest regarding the publication of this paper.

\section{References}

[1] Rock, J.A., Katayama, K.P., Martin, E.J., Woodruff, J.D. and Jones Jr., H.W. (1978) 
Factors Influencing the Success of Salpingostomy Techniques for Distal Fimbrial Obstruction. Obstetrics \& Gynecology, 52, 591.

[2] Baudet, J.H. and Segui, B. (1981) Stérilité d’origine tubaire. Révision accélérée en gynécologie. Maloine, Paris, 168-181.

[3] Dchanet, C., Flandrin, A., Reyftmann, L., Hamamah, S., Hedon, B. and Dechaud, H. (2007) Chirurgie de la stérilité tubaire distale. EMC (Elsevier Masson SAS, Paris), Techniques chirurgicales-Gynécologie, 41-527. https://doi.org/10.1016/S1624-5857(07)73023-5

[4] Tsachoua, C.L.B. (2006) Endoscopic Surgery in the Diagnosis and the Management of Female Sterilities. Medicine Thesis, Bamako, No. 57.

[5] Jeffrey, B.R., Alan, H.D., Neri, L., Mary, L.P., Frederick, N. and Phil, D. (1986) Neosalpingostomy: Comparison of 24- and 72-Month Follow-Up Time Shows Increased Pregnancy Rate. Fertility and Sterility, 45, 296-298. https://doi.org/10.1016/S0015-0282(16)49171-5

[6] Alan, G. (1971) Infertility Surgery of the Oviduct. Fertility and Sterility, 22, 496-503.

[7] Choe, J. and Check, J.H. (1999) Salpingectomy for Unilateral Hydrosalpinx May Improve In Vivo Fecundity. Gynecologic and Obstetric Investigation, 48, 285-287. https://doi.org/10.1159/000010201

[8] Al-Badawi, I.A., Fluker, M.R. and Bebbington, M.W. (199) Diagnostic Laparoscopy in Infertile Women with Normal Hysterosalpingograms. Journal of Reproductive Medicine, 44, 953-957

[9] Kaouther, D., Anissa, G., Mohamed, B.C., Ezzeddine, S. and Dalenda, C. (2014) Results of Tubal Plasty: Tunisian Study. Pan African Medical Journal, 18, 58.

[10] Boer-Meisel, M.E., Velde, E.R., Habbema, J.D. and Kardaun, J.W. (1986) Predicting the Pregnancy Outcome in Patients Treated for Hydrosalpinx: A Prospective Study. Fertility and Sterility, 45, 23-29. https://doi.org/10.1016/S0015-0282(16)49091-6

[11] Gomel, V. and McComb, P. (1981) Unexpected Pregnancies in Women Amlicted by occlusive Tubal Disease. Fertility and Sterility, 36, 529. https://doi.org/10.1016/S0015-0282(16)45808-5

[12] Verhoeven, H.E., Berry, H., Frantzen, C. and Schlosser, H.W. (1983) Surgical Treatment of Distal Tubal Occlusions: A Review of 143 Cases. Journal of Reproductive Medicine, 28, 293.

[13] Sanogo, Z., Koïta, A.K., Keita, S., Soumare, L., Fogang, K.J.J., Sacko, O., Koumare, S., Camara, M., Camara, A., Sima, M., Goita, D., Togola, B. and Sangare, D. (2014) Contribution of Endoscopic Neosalpingostomy in the Management of Tubal Infertility. Journal de Coeliochirurgie, 91, 1-5.

[14] Margit, E.B.M., Egbert, R.V.T.E., Dik, F.H.J. and Jan, W.P.F.K. (1986) Predicting the Pregnancy Outcome in Patients Treated for Hydrosalpinx: A Prospective Study. Fertility and Sterility, 45, 23-29. https://doi.org/10.1016/S0015-0282(16)49091-6

[15] DeCherney, A.H. and Kase, N. (1981)' A Comparison of Treatment for Bilateral Fimbrial Occlusion. Fertility and Sterility, 35, 162. https://doi.org/10.1016/S0015-0282(16)45316-1

[16] Vasquez, G., Boeckx, W., Winston, R.M.L., et al. (1980) Human Tubal Mucosa and Reconstructive Surgery. In: Crosignani, P.G. and Rubin, B.L., Eds., Microsurgery in Female Infertility, Academic Press, New York, 41.

[17] Shirodkar, V.N. (1966) Factors Influencing the Results of Salpingostomy. International Journal of Fertility, 11, 361-365.

[18] Caspi, E., Halperin, Y. and Bukovsky, I. (1979) The Importance of Periadnexal Ad- 
hesions in Tubal Reconstructive Surgery for Infertility. Fertility and Sterility, 31, 296-300. https://doi.org/10.1016/S0015-0282(16)43877-X

[19] Jennifer, L.T., Tiffany, J.W. and Carolyn, B.C. (1986) Repeat Tuboplasty Compared with Primary Microsurgery for Post Inflammatory Tubal Disease. Fertility and Sterility, 45, 784-787. https://doi.org/10.1016/S0015-0282(16)49393-3

[20] Togas, T. (1986) Salpingo-Ovariolysis: A Comparison between Laser Surgery and Electrosurgery. Fertility and Sterility, 45, 489-491. https://doi.org/10.1016/S0015-0282(16)49274-5

[21] Hulka, J.F., Omran, K. and Berger, G.S. (1978) Classification of Adnexal Adhesions: A Proposal and Evaluation of Its Prognostic Value. Fertility and Sterility, 30, 661-665. https://doi.org/10.1016/S0015-0282(16)43693-9

[22] Gomel, V. and Swolin, K. (1980) Salpingostomy: Microsurgical Technique and Results. Clinical Obstetrics and Gynecology, 23, 1243-1258. https://doi.org/10.1097/00003081-198012000-00029

[23] Donnez, J. and Casanas-Roux, F. (1986) Prognostic Factors Influencing the Pregnancy Rate after Microsurgical Cornual Anastomosis. Fertility and Sterility, 46, 1089-1092. https://doi.org/10.1016/S0015-0282(16)49885-7

[24] Gomel, V. (1980) Classification of Operations for Tubal and Peritoneal Factors Causing Infertility. Clinical Obstetrics and Gynecology, 23, 1259-1260.

https://doi.org/10.1097/00003081-198012000-00030 